\title{
Hypothyroidism in type 2 diabetics - a hospital-based prevalence study
}

\author{
Kiran Narukurthi R. ${ }^{1}$, Radha Mani C. ${ }^{2 *}$, Ala D. ${ }^{3}$, Chandra T. ${ }^{4}$ \\ DOI: https://doi.org/10.17511/ijmrr.2020.i01.16 \\ ${ }^{1}$ Ravi Kiran Narukurthi, Associate Professor, Department of General Medicine, GSL Medical College, Rajahmundry, Andhra Pradesh, India. \\ 2* C (Mandava) Radha Mani, Associate Professor, Department of General Medicine, GSL Medical College, Rajahmundry, Andhra Pradesh, \\ India. \\ 3 Deepthi Ala, Resident, Department of General Medicine, GSL Medical College, Rajahmundry, Andhra Pradesh, India. \\ 4 T Jaya Chandra, Scientist Incharge, Central Research Laboratory, GSL Medical College, Rajahmundry, Andhra Pradesh, India.
}

Introduction: Thyroid hormones and insulin are the antagonists and both are involved in cellular metabolism. With this, the study was planned to find the prevalence of thyroid dysfunction in type 2 diabetes mellitus (DM). Materials and Methods: Study was conducted in the department of General Medicine, GSL Medical College. The study protocol was approved by the institutional ethical committee. All type 2 diabetics aged $>30 y r s$, irrespective of treatment were included in the study. Individuals on metformin therapy, smokers, thyroid hormone users, who underwent thyroid surgery, individuals on radioiodine therapy, pregnant women and individuals on steroids were excluded. Venous blood samples were taken from for fasting blood glucose and 2-hour post glucose blood sugar, glycosylated hemoglobin, lipid profile, and thyroid function estimated measured by using the autoanalyzer. Chi-square test was used to compare differences in categorical variables and $p<0.05$ was considered statistically significant. Results: Among 104 participants, 82.7\% were euthyroid, $12.5 \%$ had subclinical and $4.8 \%$ had clinical hypothyroidism; statistically the difference not significant when age/gender was correlated. But there was a significant association with dyslipidemia. Conclusion: Hypothyroidism is very common among type 2 DM individuals and both complement others' metabolism.

Keywords: Diabetes, Thyroid, Thyroidism, Individual

Corresponding Author

C (Mandava) Radha Mani, Associate Professor, Department of General Medicine, GSL Medical College, Rajahmundry, Andhra Pradesh, India. Email: gslcentralresearchlab@gmail.com
How to Cite this Article

Narukurthi RK, (Mandava) Mani CR, Ala D, Chandra TJ. Hypothyroidism in type 2 diabetics - a hospitalbased prevalence study. Int J Med Res Rev. 2020;8(1):105-109.

Available From

https://ijmrr.medresearch.in/index.php/ijmrr/article/ view/1152
To Browse

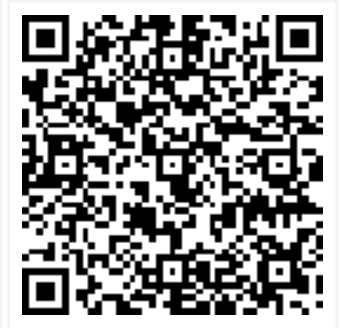

Manuscript Received 2020-02-02

Conflict of Interest No 2020-02-12

Funding

Nil
Review Round 2 2020-02-17

Ethical Approval Yes
Review Round 3

Plagiarism X-checker $6 \%$
Accepted 2020-02-22 (c) 2020 by Ravi Kiran Narukurthi, C (Mandava) Radha Mani, Deepthi Ala, T Jaya Chandra and Published by Siddharth Health
Research and Social Welfare Society. This is an Open Access article licensed under a Creative Commons Attribution 4.0 International License https://creativecommons.org/licenses/by/4.0/ unported [CC BY 4.0]. 


\section{Introduction}

Diabetes mellitus (DM), most common endocrine disorder, characterized by persistent hyperglycemia resulting from defects in insulin secretion, insulin action, or both. Development of diabetes involves several pathogenic processes ranging from autoimmune destruction of the $\beta$ cells of the pancreas with consequent insulin deficiency to abnormalities that result in resistance to insulin action [1]. Hyperglycemia is the principal cause of diabetic complications. Sedentary lifestyle, various diet patterns, ethnicity, and genetic predisposition are the major factors responsible for the causes of the epidemic [2]. Thyroid disorders are also common in the general population and it is the second most common endocrine disorder. As a result, it is common for an individual to be affected by both thyroid disease and diabetes [3]. Various studies have reported the low prevalence of thyroid dysfunction among diabetic patients, between 2.2 to $17 \%$ in their respective populations $[4,5]$. However, few studies have shown a higher prevalence of thyroid dysfunction in diabetes from $31 \%$ to $46.5 \%$ $[6,7]$. Thyroid hormones and insulin are the antagonists and both are involved in cellular metabolism of carbohydrates, proteins, and lipids. The functional impairment occurs in thyroid hormone as well as insulin if their levels changed [8]. DM appears to influence thyroid function in two sites; firstly, at the level of hypothalamic control of $\mathrm{TSH}$ release and secondly at the conversion of T4 to T3 in the peripheral tissues. Hyperglycemia causes reversible reduction of the activity and hepatic concentration of T4 5'deiodinase, low serum T3, increase in reverse $\mathrm{T} 3$ and also variation in the level of T4 [9]. The recognition of this interdependent relationship between thyroid disease and diabetes is of importance to guide clinicians on the optimal management of both these conditions. With this, the study was planned to find the prevalence of thyroid dysfunction in type 2 DM.

\section{Materials and Methods}

Settings: Study was conducted in the department of General Medicine, GSL Medical College.

Duration of study: The study was conducted from November 2015 to April 2017.

Sampling method: Random sampling was considered in this study.

Inclusion criteria: All type 2 diabetics aged $>30$
Yrs, irrespective of treatment were included in the study.

Exclusion criteria: Individuals on metformin therapy, smokers, thyroid hormones users, who underwent thyroid surgery, individuals on radioiodine therapy, pregnant women and individuals on steroids were excluded.

Sample size: All the individuals who satisfy the inclusion criteria during the study period were included in the study.

Ethical approval: Study protocol was approved by the institutional ethical committee.

A pre-structured questionnaire was used to collect clinical data. Baseline data including age, detailed medical history, past history, family history, drug history, and personal history were recorded. Clinical examination, routine and relevant investigations were carried out for all participants. Diagnosis of diabetes was made according to the American Diabetic Association criteria or if the subjects were already taking diabetic drugs. Study Subjects were investigated for diabetic complications like retinopathy, ischemic heart disease, nephropathy. Venous blood samples were taken from an antecubital vein of the arm by means of clean venipuncture after an overnight fast for fasting blood glucose and 2-hour post glucose blood sugar, glycosylated hemoglobin, and lipid profile. Venous blood also assayed for thyroid function (T3, T4, and $\mathrm{TSH})$. Plasma glucose concentration was estimated using the GOD- POD (Glucose oxidase - Peroxidase) method and thyroid hormones were estimated using principle combining a one-step enzyme immunoassay. TSH levels are $<10 \mathrm{mIU} / \mathrm{L}$ was considered subclinical hypothyroidism and $>10 \mathrm{mIU} / \mathrm{L}$ is clinical hypothyroidism. Serum lipids (total cholesterol, triglycerides, LDL cholesterol, and HDL plasma cholesterol concentrations) were measured by using the autoanalyzer. Chi-square test was used to compare differences in categorical variables and $\mathrm{p}<0.05$ was considered statistically significant.

\section{Results}

A total of 104 participants with DM were included in the study. Among these $82.7 \%$ were euthyroid, $12.5 \%$ had subclinical and $4.8 \%$ had clinical hypothyroidism; statistically the difference not significant when age/gender was correlated with hypothyroidism in type 2 diabetics. There was a significant association between dyslipidemia and 
Hypothyroidism in type 2 diabetics. Age-wise, in euthyroid category, $4.8 \%$ (5) were in $30-40$ years group, $27 \%$ (28) in $41-50$ years group, $28.8 \%$ (30) in 51-60 years group, $20 \%$ (21) in $61-70$ years group and $1.9 \%$ (2) in $>71$ years group. In the subclinical category, $1.9 \%$ (2) were in the $30-40$ years group, $4.8 \%$ (5) in $41-50$ years group, $3.8 \%$ (4) in 51-60 years group, $1.9 \%$ (2) in $61-70$ years group. In the clinical category, $9 \%$ (1) in the $41-50$ years group, $1.9 \%$ (2) in the $51-60$ years group, $1.9 \%$ (2) in $61-70$ years group. Statistically, there was no significant difference (Table 1 ).

Table-1: Age-wise thyroid function results among type 2 diabetics; $n$ (\%).

\begin{tabular}{|l|l|l|l|l|}
\hline \multicolumn{1}{|c|}{ Age } & \multicolumn{1}{|c|}{ Euthyroid } & \multicolumn{1}{|c|}{ Subclinical } & \multicolumn{1}{c|}{ Clinical } & \multicolumn{1}{|c|}{ Total } \\
\hline $30-40$ & $5(4.8)$ & $2(1.9)$ & 0 & $7(6.7)$ \\
\hline $41-50$ & $28(27)$ & $5(4.8)$ & $1(0.9)$ & $34(32.7)$ \\
\hline $51-60$ & $30(28.8)$ & $4(3.8)$ & $2(1.9)$ & $36(34.6)$ \\
\hline $61-70$ & $21(20)$ & $2(1.9)$ & $2(1.9)$ & $25(24)$ \\
\hline$>71$ & $2(1.9)$ & 0 & 0 & $2(1.9)$ \\
\hline Total & $86(83.3)$ & $13(12.5)$ & $5(4.8)$ & $104(100)$ \\
\hline
\end{tabular}

Gender wise, in males, 32.6\% (34) were euthyroid, $5.8 \%$ (6) were subclinical and $1.9 \%$ (2) were clinical. In the female, 50\% (52) were euthyroid, $6.7 \%$ (7) were subclinical and $2.9 \%$ (3) were clinical. Statistically, there was no significant difference (Table 2).

Table-2: Gender wise thyroid function results among type 2 diabetics; $\mathbf{n}(\%)$.

\begin{tabular}{|l|l|l|l|l|}
\hline \multicolumn{1}{|c|}{ Age } & \multicolumn{1}{c|}{ Euthyroid } & \multicolumn{1}{c|}{ Subclinical } & \multicolumn{1}{c|}{ Clinical } & \multicolumn{1}{c|}{ Total } \\
\hline Male & $34(32.6)$ & $6(5.8)$ & $2(1.9)$ & $42(40.4)$ \\
\hline Female & $52(50)$ & $7(6.7)$ & $3(2.9)$ & $62(59.6)$ \\
\hline Total & $86(83.3)$ & $13(12.5)$ & $5(4.8)$ & $104(100)$ \\
\hline Chi-Square: $0.206 ;$ df: $2, \mathrm{P}=0.902 ;$ statistically not significant \\
\hline
\end{tabular}

When thyroid test results were correlated with dyslipidemia, in euthyroid, 32\% DM had dyslipidemia; Dyslipidemia was resent in $12.5 \%$ and $4.8 \%$ cases respectively in subclinical and clinical cases. Whereas in $51 \%$ of cases dyslipidemia was not identified. Statistically, there was a significant difference (Table 3).

Table-3: Thyroid function results among type 2 diabetics with dyslipidemia; $\mathbf{n}(\%)$.

\begin{tabular}{|l|l|l|l|l|}
\hline \multicolumn{1}{|c|}{ Dyslipidemia } & \multicolumn{1}{|c|}{ Euthyroid } & \multicolumn{1}{|c|}{ Subclinical } & \multicolumn{1}{c|}{ Clinical } & \multicolumn{1}{|c|}{ Total } \\
\hline Present & $33(32)$ & $13(12.5)$ & $5(4.8)$ & $51(49)$ \\
\hline Absent & $53(51)$ & 0 & 0 & $53(51)$ \\
\hline Total & $86(83.3)$ & $13(12.5)$ & $5(4.8)$ & $104(100)$ \\
\hline Chi-Square: $22.621 ;$ df: $2, \mathrm{P}=0.0001 ;$ statistically significant \\
\hline
\end{tabular}

\section{Discussion}

Diabetes is a leading cause of morbidity and mortality worldwide. Its incidence is increasing day by day all over the world, posing a major threat to public health. The influence of endocrine and nonendocrine organs other than the pancreas on diabetes mellitus is documented. Thyroid disorders are also very common endocrine disorders in the general population. Hence it is common for an individual to be affected by both thyroid diseases and diabetes. The incidence is increasing day by day all over the world, posing a major threat to public health. The major alterations in the thyroid hormone system are a reduction in the TSH stimulation of the thyroid gland, probably caused by central hypothyroidism, and in the peripheral generation of T3 from T4 [4,10]. Unrecognized thyroid dysfunction may worsen metabolic control and impede the management of diabetes. Therefore, diabetic patients need to be screened for thyroid dysfunction [11]. The study examined the prevalence of subclinical, clinical hypothyroidism in subjects with type 2 diabetics and also examined how the duration of diabetes affects the prevalence of hypothyroidism in type 2 diabetics along with the emphasis on glycemic control and on long term complications. In the present study, out of 104 types 2 diabetic patients, the prevalence of hypothyroidism was more common in the age group of 41-70 years. This indicates that the peak incidence was in the 4th and 5th decades of life. In spite of this prevalence, there was no statistical significance difference between age and hypothyroidism (Table $1 ; P>0.05$ ). In one retrospective study hypothyroidism was reported to be quite high in type 2 DM patients with $>45$ years [12]. Telwani AA et al. concluded that hypothyroidism was more common in the age group $>50$ years [1]. Thyroid hormones exert profound effects on the regulation of glucose homeostasis. These effects include modifications of circulating insulin levels and counter-regulatory hormones, intestinal absorption, hepatic production and peripheral tissues uptake of glucose [13]. Gender wise, in this study, thyroid defect was reported in $7.7 \%$ and $9.6 \%$, respectively in male and female; statistically there was no significant difference (Table 2; P>0.05). A study done by Udiong CEJ. reported that TSH levels in diabetics were significantly lower than the level in non-diabetics and male diabetics had lower levels of TSH [6]. In another report from India also reported a high 
Incidence of thyroid disorder among females; $36 \%$, $22 \%$, respectively and elder population with $>60$ years, had more incidence of thyroid disorder [14]. Epidemiological studies reported $2-4 \%, 4-20 \%$ of overt hypothyroidism and subclinical hypothyroidism range respectively and higher incidence among women with $>60$ years age $[15,16]$. Hypothyroidism or thyroid overactivity is most frequently induced by an autoimmune process. It was mentioned that the number of people with thyroid disorders in India exceeds 40 million [17]. Various studies in the recent past have revealed a higher incidence of thyroid dysfunction in diabetics compared to the normal population. In population studies, overt hypothyroidism or its subclinical manifestations range between $2-4 \%$ and $4-20 \%$ respectively both being significantly higher in women above the age of 60 years [16]. Hypothyroidism is a clinical state due to the decreased secretion of thyroid hormones viz., thyroxine and triiodothyronine or very rarely due to the decreased activity of these hormones at tissue levels. The term 'subclinical hypothyroidism' is used to define that grade of primary hypothyroidism in which there is an elevated thyroid-stimulating hormone concentration in the presence of normal serum free thyroxine and triiodothyronine concentrations. Subclinical hypothyroidism may progress to overt hypothyroidism in approximately 2-5\% cases annually. All patients with overt hypothyroidism and subclinical hypothyroidism with $\mathrm{TSH}>10 \mathrm{mIU} / \mathrm{L}$ should be treated [18]. Hyperthyroidism leads to lipid peroxidation whereas hypothyroidism causes diminished glucose oxidation. LDL clearance leads to lowered cholesterol and triglyceride levels. It has been reported that $\mathrm{T} 3$ enhances fasting plasma glucose and free fatty acid levels [19]. In the present study, dyslipidemia was identified in 32\%, 12.5\%, 4.8\% participants, respectively in euthyroid, subclinical and clinical hypothyroid patients, statistically, the difference was significant (Table 3; $\mathrm{P}<0.05$ ). Literature also reported a high percentage of severe clinical hypothyroidism (SCH) [20]. These changes might indicate that elevated $\mathrm{TSH}$ can affect blood lipids and vascular endothelium in patients with Type 2 diabetes, although whether $\mathrm{SCH}$ is the primary reason for the high prevalence of CHD remains controversial. Whereas dyslipidemia was reported to be $95.5 \%$ among the type 2 DM in one cross-sectional report $[21,22,23]$.

\section{Conclusion}

With these study findings and the available kinds of literature, hypothyroidism is very common among type 2 DM individuals. However, thyroid hormones also exert profound effects in the regulation of glucose homeostasis prolonged peripheral glucose accumulation, gluconeogenesis, diminished hepatic glucose output and reduced disposal of glucose. So it is very clear that both can complement other metabolism.

\section{Limitation}

Small sample size is the major limitation of the study.

\section{What does the study add to the existing knowledge}

Hypothyroidism is very common among type 2 DM individuals. Most of the cases are euthyroid and gender-wise, more common among females. This is increased with the age of the individual.

\section{Author's contribution}

Dr. Ravi Kiran Narukurthi: Study design, Literature survey, data analysis, paper writing. Dr. C (Mandava) Radha Mani: Study design, paper writing. Dr. Deepthi Ala: Main work, Literature survey, data analysis. Dr. T Jaya Chandra: Data analysis, paper writing, statistical part.

\section{Reference}

01. Ajaz Ahmad Telwani, Zahid Hussain Wani, Younis Ashraf, Aejaz Ahmad Shah. Prevalence of thyroid dysfunction in type 2 diabetes mellitusa case control study. Int J Res Med Sci. 2017;5(10)4527-4531.

doi: [Article] [Crossref]

02. American diabetes association. Diagnosis and classification of diabetes mellitus. Diabetes care. 2010;33(1)562-569.

doi: [Article] [Crossref]

03. Gray RS, Irvine WJ, Clarke BF. Screening for thyroid dysfunction in diabetics. $\mathrm{Br}$ Med $\mathrm{J}$. $1979 ; 2(6202) 1439$.

doi: [Article] [Crossref] 
04. Perros $P$, McCrimmon RJ, Shaw G, Frier BM. Frequency of thyroid dysfunction in diabetic patients- value of annual screening. Diabet Med. $1995 ; 12(7) 622-627$.

doi: [Article] [Crossref]

05. Smithson MJ. Screening for thyroid dysfunction in a community population of diabetic patients. Diabet Med. 1998;15(2)148-150.

doi: [Article] [Crossref]

06. Udiong $\mathrm{CEJ}$, Udoh $\mathrm{AE}$, Etukudoh. Evaluation of thyroid function in diabetes mellitus in Calabar, Nigeria. Ind J Clin Biochem. 2007;22;74-78. doi: [Article] [Crossref]

07. Celani MF, Bonati ME, Stucci N. Prevalence of abnormal thyrotropin concentrations measured by a sensitive assay in patients with Type 2 diabetes mellitus. Diabete Res. 1994;27(1)1525.

[Crossref]

08. Sugure DD, McEvoy M, Drury MI. Thyroid disease in diabetics. Postgrad Med J. 1999;91(1)680-684.

doi: [Article] [Crossref]

09. Shah SN. Thyroid disease in diabetes mellitus. J Assoc Physicians India. 1984;32(12)1057-1059. [Crossref]

10. Papazafiropoulou A, Sotiropoulos A, Kokolaki A, Kardara M, Stamataki P, Pappas S. Prevalence of thyroid dysfunction among Greek type 2 diabetic patients attending an outpatient clinic. J Clin Med Res. 2010;2(2)75- 78.

doi: [Article] [Crossref]

11. Chen HS, Wu TE, Jap TS, Lu RA, Wang ML, Chen $R L$, Lin HD. Subclinical hypothyroidism is a risk factor for nephropathy and cardiovascular diseases in type 2 diabetic patients. Diabet Med. 2007;24(12)1336-1344.

doi: [Article] [Crossref]

12. Demitrost $L$, Ranabir S. Thyroid dysfunction in type 2 diabetes mellitus- A retrospective study. Indian J Endocrinol Metab. 2012;16(2)S334-5. doi: [Article] [Crossref]

13. Brenta G. Diabetes and thyroid disorders. Brit J Diab Vascul Dis. 2010;10(4)172-177. doi: [Article] [Crossref]

14. Ravishankar SN, Champakamalini, Venkatesh, Mohsin. A prospective study of thyroid dysfunction in patients with Type 2 diabetes in general population. Arch Med. 2013;5(1-2)1-5. [Crossref]
15. Duntas LH, OrgiazziJ, Brabant G. The interface between thyroid and diabetes mellitus. Clin Endocrinol. 2011;75(1)1-9.

doi: [Article] [Crossref]

16. Nobre EL, Jorge Z, Pratas S, Silva C, Castro JJ. Profile of the thyroid function in a population with type 2 diabetes mellitus. Proceed Joint Brit Endocrine Soc. 2002;298.

[Crossref]

17. Vij V, Chitnis P, Gupta VK. Evaluation of Thyroid Dysfunction among Type 2 Diabetic patients. Int J Pharma Biol Sci. 2012;2(4)150-155.

[Crossref]

18. Khandelwal, Tandon. Overt and subclinical hypothyroidism, who to treat and how, department of endocrinology and metabolism, All India Institute of Medical Sciences, New Delhi, India. Drugs. 2012;72(1)17-33. doi: [Article] [Crossref]

19. Wang C. The relationship between type 2 diabetes mellitus and related thyroid diseases. J Diabetes Res. 2013;10;1-12. doi: [Article] [Crossref]

20. Keyur Brahme, Jaydeep Devaliya, Ishani Haldar, Mahendra Parmar, Kalpita Shringarpure. Does the glycemic control and duration of diabetes affect Thyroid Dysfunction? A cross-sectional study from a tertiary hospital in Central Gujarat. Sch J App Med Sci. 2016;4(8D)3031-3036. doi: [Article] [Crossref]

21. GA Mohamed, AM Elsayed. Subclinical hypothyroidism ups the risk of vascular complications in type 2 diabetes. Alexandria J Med. 2017;53(3)285-288.

[Crossref]

22. Mouradian M, Abourizk N. Diabetes mellitus and thyroid disease. Diabetes Care. 1983;6(5)512520. doi: [Article] [Crossref]

23. Brenta G. A View of Diabetes from the Thyroid Corner. Thyroid Int. 2011;3;3-11. [Crossref] 